\title{
Laser linewidth requirements and improvements for coherent optical beam forming networks in satellites
}

\author{
Gliese, Ulrik Bo; Christensen, Erik Lintz; Stubkjær, Kristian
}

Published in:

Journal of Lightwave Technology

Link to article, DOI:

$10.1109 / 50.81982$

Publication date:

1991

Document Version

Publisher's PDF, also known as Version of record

Link back to DTU Orbit

Citation $(A P A)$ :

Gliese, U. B., Christensen, E. L., \& Stubkjær, K. (1991). Laser linewidth requirements and improvements for coherent optical beam forming networks in satellites. Journal of Lightwave Technology, 9(6), 779-790. https://doi.org/10.1109/50.81982

\section{General rights}

Copyright and moral rights for the publications made accessible in the public portal are retained by the authors and/or other copyright owners and it is a condition of accessing publications that users recognise and abide by the legal requirements associated with these rights.

- Users may download and print one copy of any publication from the public portal for the purpose of private study or research.

- You may not further distribute the material or use it for any profit-making activity or commercial gain

- You may freely distribute the URL identifying the publication in the public portal 


\title{
Laser Linewidth Requirements and Improvements for Coherent Optical Beam Forming Networks in Satellites
}

\author{
Ulrik Gliese, Erik Lintz Christensen, and Kristian E. Stubkjær, Member, IEEE
}

\begin{abstract}
A coherent optical beam forming network principle using phase-locked semiconductor lasers as sources is presented. For this scheme the spectral purity of the intermediate frequency derived from mixing the signals from two semiconductor lasers is considered and the relationship between the linewidths of the lasers in a satellite transmitter and the phase error at the detector of a microwave differential quaternary phase-shift keying earth station receiver is analyzed. The demands placed on the linewidths from the point of view of phase stability requirements are calculated for coherent optical beam forming networks using quaternary phase-shift keying modulation at data rates of 33 and $131 \mathrm{Mb} / \mathrm{s}$ as examples. Furthermore, it is shown that a substantial improvement in performance can be achieved when phase locking the two lasers to each other is feasible.
\end{abstract}

\section{INTRODUCTION}

$F_{n}$ UTURE satellite antennas are required to offer a large number of shaped, reconfigurable, and repositionable beams for various earth stations and mobile receivers. These antennas might be implemented as active phased array antennas based on a large number of active microwave array elements. Unfortunately such antennas tend to be bulky and costly but as the microwave monolithic integrated circuits (MMIC) technology develops, these problems will be greatly reduced.

The patterns of the radiated antenna beams are determined by the amplitude and the phase of the current in each antenna element. Therefore, it is necessary to use some sort of beam forming network which can feed each antenna subarray element individually with the proper amplitude and phase. This type of signal distribution is a challenging task and conventional feeds using waveguides or coaxial cables will not be feasible for antennas with a large number of beams. Waveguides offer low loss and good phase accuracy but they are bulky and heavy. Coaxial cables are more flexible and fairly lightweight but they have high insertion losses and poor phase accuracy compared to the waveguides. By contrast fiber-optic links offer an attractive solution for the distribution of the sig-

Manuscript received September 10, 1990; revised February 13, 1991

The authors are with the Center for Broadband Telecommunications Electromagnetics Institute. Technical University of Denmark, DK-2800 Lyngby, Denmark.

IEEE Log Number 9144426 nals in a phased array antenna system. Optical fibers are small, flexible, lightweight, and practically immune to electromagnetic interference. Furthermore, they have negligible losses, good phase accuracy, and very large bandwidths. Amplitude accuracy in a fiber-optic network will be dependent on the accuracy of the coupling ratios. Therefore, the fiber couplers in the network must be carefully selected.

The functions of the fiber-optic distribution network are: distribution and transmission of the communication signal, distribution of the phase and frequency reference, and beam control. These functions can all be achieved in one fiber-optic network; an optical beam forming network (OBFN). Various experiments consider fiber-optic distribution links by direct detection methods [1]-[3], OBFN's by direct detection methods [4]-[6], and OBFN's by coherent methods using injection locked lasers [7]-[10] or frequency shifting [11]. For direct detection techniques, the lasers employed need to have a modulation bandwidth corresponding to the microwave frequency of the comsunication signal carrier (here $12.5 \mathrm{GHz}$ ). This is very difficult to obtain especially when high phase accuracy is required.

Coherent techniques seem attractive for a number of reasons. It is possible to obtain a better carrier to noise $(\mathrm{C} / \mathrm{N})$ performance with coherent schemes than with direct detection schemes. This results in a better power budget which allows for more arrays or fewer laser transmitter modules. Furthermore, the generated microwave frequency at the antenna can easily be tuned by simply tuning one of the two lasers employed. Finally, coherent techniques give greater possibilities for optical control and optical processing.

The paper focuses on one of the important constraints, namely the laser linewidth and the paper is outlined as follows. In Section II the principle of the coherent OBFN is described together with power budget calculations for the network. The requirements for laser linewidth are derived in Section III which leads to Section IV where the improvements obtained by using a phase locked loop are analyzed. In Section V limitations on the implementation of phase locked loops are considered. Finally in Section VI, some conclusions are drawn. 


\section{Coherent Optical Beam Forming}

The basic idea behind coherent optical beam forming is the generation of the microwave carrier by the beating of two lasers. The microwave signal phase stability requirements are very high since the signals must comply with existing microwave earth station receivers. As an example, the requirements in a microwave differential quaternary phase-shift keying (DQPSK) receiver is a maximum $\mathrm{rms}$ differential phase error $\sigma_{\Delta \phi}$ of $2.8^{\circ}$ [12]. To generate such a stable microwave carrier by beating two coherent optical sources, it is necessary to use very stable lasers. The requirements placed on the laser linewidths will be derived in this paper and it will be shown that stabilization techniques have to be used unless very narrow linewidth lasers are used.

The lasers of interest for a satellite-based system are $\mathrm{Nd}$ : YAG lasers, external cavity lasers, and distributed feedback (DFB) semiconductor lasers or the like. The two first types of lasers have very narrow linewidths and the stabilization requirements are not as stringent as for DFB lasers. On the other hand, they consume more power and are larger in size than DFB lasers. Furthermore, external cavity lasers require high mechanical stability, a condition which is difficult to fulfill in satellites. Finally, DFB lasers also have the advantage of potential optoelectronic integration.

For stabilization of semiconductor lasers, either optical techniques, i.e., injection locking [7]-[10] or electronic techniques, i.e., frequency or phase locking [13]-[15] can be applied. Optical techniques, however, are likely to be the less reliable of the two due to problems with mechanical stability.

We propose a coherent OBFN using phase-locked DFB lasers as the optical sources. A schematic diagram of the coherent OBFN is given in Fig. 1. For simplicity the configuration shown considers only the transmission of one antenna beam. The coherent OBFN can be divided into four main modules.

1) The transmitter module, which supplies the optical signals, consists of two phase-locked DFB lasers. The phase-locked loop (PLL) is a heterodyne optical PLL, i.e., the two DFB lasers are locked to a reference microwave source oscillating at the microwave carrier frequency. This ensures that the beat frequency of the two lasers is fixed at the microwave carrier frequency. Furthermore, the PLL greatly reduces the phase noise of the laser beat signal so that a phase stable microwave signal at the antenna is obtained.

2) The modulator module, where the transmitter laser signal is fed to an optical phase modulator which is driven by the data input signal. The modulation scheme can be QPSK modulation, e.g., at bit rates of 33 or $131 \mathrm{Mb} / \mathrm{s}$ which are two of the formats used in microwave satellite links.

3) The beam forming network, where both the modulated transmitter laser signal and the signal from the volt-

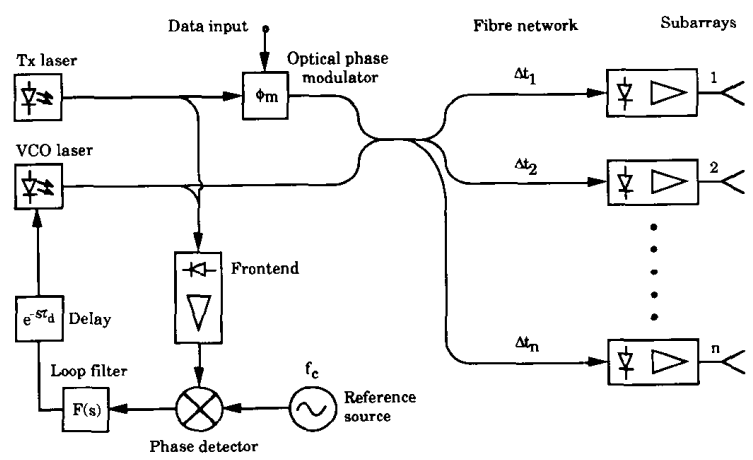

Fig. 1. Schematic of a coherent optical beam forming network (OBFN) consisting of an optical phase-locked transmitter module, an optical phase modulator, a fiber-optic distribution network, and an active phased array antenna with $n$ subarrays.

age controlled oscillator (VCO) laser are distributed to the antenna elements by the fiber-optic distribution network. Beam forming and beam steering are accomplished optically in the fiber network. The phase of the microwave signals at the antenna elements is controlled by applying the proper delays $\left(\Delta t_{1}, \Delta t_{2}, \cdots, \Delta t_{n}\right)$ individually in all the fiber arms. This can either be a static adjustment whereby the fibers are adjusted to specific lengths giving true time delay (TTD) [5], [16] or a variable microwave phase adjustment by the use of variable optical delays. Proposals for such variable optical delays have been presented [4], [9]-[11]. However, the implementation of variable optical delays with sufficient delay is very difficult. Therefore, the proposed OBFN architecture is limited to static beams until useful variable optical delays are realized.

4) The active phased array antenna consists of $n$ subarrays from which the microwave communication signal is radiated. Each subarray consists of a front end, an amplifier chain, and a number of radiating array elements. The front ends are based on $\mathrm{p}-\mathrm{i}-\mathrm{n}$ photodiodes since they are faster than Avalanche photodiodes (APD's). The phase and amplitude regulated transmitter laser and VCO laser optical signals are detected with the front ends in each subarray thereby generating the modulated microwave signal as the beat signal of the two optical signals.

For the coherent detection method, the polarizations of the two optical signals have to be alike. That can be ensured by the use of polarization preserving fibers and optical components or by the use of polarization controllers. It is also possible to use more advanced receivers such as polarization diversity receivers but at the price of far more complex electronics at the antenna interface which is located in a harsh environment.

The number of subarrays that can be fed from one transmitter module is determined by the laser's optical output power, the laser's relative intensity noise (RIN), and the thermal noise of the front ends. Subsequently, we 
have derived the maximum number of subarrays per transmitter module as

$$
n=2^{\left(P_{\text {out }}-P\right) / 3 \mathrm{~dB}}
$$

where $P_{\text {out }}$ is the output power (in $\mathrm{dBm}$ ) from each laser at the entry of the fiber network which is assumed to be implemented with 3 -dB couplers. $P$ is the received power (in $\mathrm{dBm}$ from each laser) required at the detectors to obtain a specific carrier-to-noise ratio at the output of the front ends. Here it is presumed that the output powers from each of the two lasers are equal. The expression for the power $P$ is derived from the carrier-to-noise density ratio $\left(\mathrm{C} / \mathrm{N}_{0}\right)$ given by

$$
\mathrm{C} / \mathrm{N}_{0}=\frac{I_{P O}^{2}}{\frac{d\left\langle i_{\mathrm{RIN}}^{2}\right\rangle}{d f}+\frac{d\left\langle i_{q}^{2}\right\rangle}{d f}+\frac{d\left\langle i_{t}^{2}\right\rangle}{d f}}
$$

where $I_{P O}^{2}$ is the detected photo current, $d\left\langle i_{\mathrm{RIN}}^{2}\right\rangle / d f$ is the equivalent input noise spectral density of the relative intensity noise, $d\left\langle i_{q}^{2}\right\rangle / d f$ is the equivalent input noise spectral density of the quantum shot noise, and $d\left\langle i_{t}^{2}\right\rangle / d f$ is the equivalent input noise spectral density of the front ends. The $C / N_{0}$ is then given by:

$$
\mathrm{C} / \mathrm{N}_{0}=\frac{2 R^{2} P^{2}}{2 R^{2} \operatorname{RIN} P^{2}+4 R q P+\frac{d\left\langle i_{t}^{2}\right\rangle}{d f}}
$$

where $R$ is the responsivity of the photodiodes, RIN is the relative intensity noise of each laser, and $q$ is the electron charge. $P$ can then be isolated from (3) as most critical aspect of the coherent OBFN is the requirements for laser stability, as will be clarified in the next section.

\section{LASER Linewidth ReQuirements}

The properties of the laser transmitter module are very important due to the strict demands on phase stability of the transmitted microwave signal. The impact of laser phase noise on the bit-error-rate (BER) performance of coherent optical communication systems has been investigated and is well understood [17]-[24]. However, the demands placed on the laser linewidths from the point of view of phase stability in a coherent OBFN has not yet been treated in the literature. To assess the influence of laser linewidth we start the derivation based on a standard microwave receiver.

\section{A. The Differential Phase Fluctuation Spectrum}

The transmitted microwave signal from the satellite is detected by a microwave communication receiver such as a DQPSK receiver using a differential phase detector with a delay $\tau$ equal to the symbol time $T$, c.f., Fig. 2 .

In this type of system the total double sideband rms differential phase error $\sigma_{\Delta \phi}$ between two symbols may not exceed $\sigma_{\Delta \phi}=2.8^{\circ}$ [12]. The reason for this stringent specification to carrier phase noise is to keep the sensitivity degradation due to phase noise at a minimum since transmitter power from a satellite is extremely expensive.

$$
P=10 \cdot \log \left(\frac{-4 \frac{C}{N_{0}} q-2 \sqrt{\left(2 \frac{C}{N_{0}} q\right)^{2}-2 \frac{d\left\langle i_{t}^{2}\right\rangle}{d f}\left(\left(\frac{C}{N_{0}}\right)^{2} \mathrm{RIN}-\frac{C}{N_{0}}\right)}}{10^{-3} \cdot 4 R\left(\frac{C}{N_{0}} \mathrm{RIN}-1\right)}\right)
$$

The maximum number of subarrays per transmitter module can now be calculated by inserting (4) into (1) and as an example, it is possible to feed 256 subarrays per transmitter module when the $\mathrm{C} / \mathrm{N}_{0}$ demand is $110 \mathrm{~dB} / \mathrm{Hz}$. For this example we use lasers with an output power of $P_{\text {out }}$ $=0 \mathrm{dBm}$ and a relative intensity noise RIN of -120 $\mathrm{dB} / \mathrm{Hz}$, photodetectors with a responsivity $R$ of 0.8 and front ends with an equivalent input noise spectral density $d\left\langle i_{t}^{2}\right\rangle / d f$ of $(10 \mathrm{pA} / \sqrt{\mathrm{Hz}})^{2}$. Furthermore, the fiber network is assumed ideal without losses. This large number of subarrays indicates that, if desirable, it is possible to feed even a large active phased array antenna by only one laser transmitter module. If a number of transmitter modules are needed they must all be locked to the same microwave reference source.

As shown above, the coherent OBFN is a very interesting and promising candidate for use as a beam forming network for active phased array antennas in satellites. The
The single sideband differential phase fluctuation spectrum $S_{\Delta \phi}(f)$ is given by [25]

$$
\begin{aligned}
S_{\Delta \phi}(f) & =2 \cdot \frac{S_{f}(f)}{f^{2}} \cdot(1-\cos 2 \pi f T) \\
& =4 \cdot \frac{S_{f}(f)}{f^{2}} \cdot \sin ^{2} \pi f T .
\end{aligned}
$$

Here $S_{f}(f)$ is the single sideband frequency fluctuation spectrum of the microwave signal generated by the lasers in the transmitter module, $f$ is the frequency offset from the carrier, and $T$ is the symbol time.

By using the relationship given in (5), a realistic shape of the spectrum $S_{f}(f)$ can be taken into account. It is known that semiconductor lasers have a non-Lorentzian line shape with a frequency fluctuation spectrum contain- 


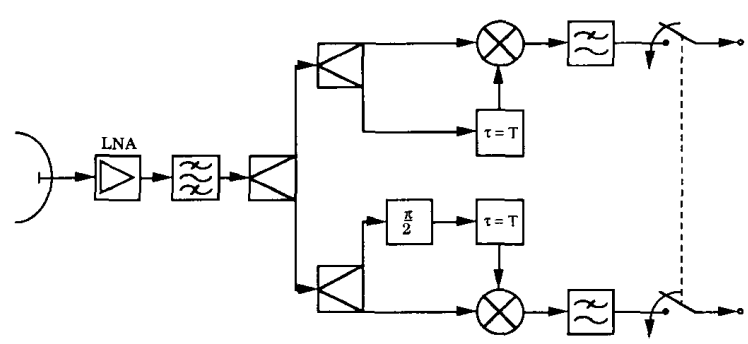

Fig. 2. DQPSK microwave receiver principle.

ing a peak at a frequency corresponding to the laser relaxation resonance frequency [26]-[29]. The influence of this high-frequency peak is determined by its position and energy. In DFB lasers biased well above threshold (as will be the case in real systems) the relaxation resonance appears at a frequency of several gigahertz and the energy of the peak is very small [28], [29]. Due to the low bit rates used in satellite links, the peak in the frequency fluctuation spectrum $S_{f}(f)$ is assumed to have no significant influence on system behavior. Therefore a Lorentzian line shape with a white frequency fluctuation spectrum is assumed and the single-sideband frequency fluctuation spectrum will then be [18]

$$
S_{f}(f)=\frac{\Delta \nu}{\pi}
$$

where $\Delta \nu$ is the $3-\mathrm{dB}$ linewidth of the microwave carrier generated by the transmitter module, i.e., the sum of the linewidths of the two lasers.

\section{B. RMS Differential Phase Error and Laser Linewidth Requirements}

By relating the rms differential phase error in the receiver to the phase noise of the lasers in the transmitter module, it is possible to calculate the maximum allowable linewidths. Knowing the spectrum from (5) and (6) makes the derivation of the total double sideband rms differential phase error $\sigma_{\Delta \phi}$ due to the lasers straightforward since the integrals may be found directly from [30]

$$
\begin{aligned}
\left(\sigma_{\Delta \phi}\right)^{2} & =\int_{0}^{\infty} S_{\Delta \phi}(f) d f \\
& =4 \int_{0}^{\infty} \Delta \nu \frac{\sin ^{2} \pi T f}{\pi f^{2}} d f \\
& =4 T \Delta \nu \int_{0}^{\infty}\left(\frac{\sin x}{x}\right)^{2} d x \\
& =2 \pi T \Delta \nu .
\end{aligned}
$$

Usually, a microwave receiver will have an IF filter in front of the demodulator (to reduce thermal noise and interference) and, furthermore, a post detection filter as an integral part of the demodulator itself (c.f. Fig. 2). The best filter, i.e., the matched filter, for detection of a square-wave modulated signal in the presence of white (thermal) noise is an integrator with an integration time equal to the symbol duration, $T$ [31]. This filter has a $\sin (x) / x$ transfer function in the frequency domain and since phase modulation is under consideration here, the phase spectrum should be multiplied with that transfer function. Consequently, the differential phase fluctuations after the filter will be

$$
\begin{aligned}
\left(\sigma_{\Delta \phi}\right)^{2} & =\int_{0}^{\infty} S_{\Delta \phi}(f) \cdot\left|H_{\phi}(f)\right|^{2} d f ; \quad H_{\phi}(f)=\frac{\sin \pi T f}{\pi T f} \\
& =4 T \Delta \nu \int_{0}^{\infty}\left(\frac{\sin x}{x}\right)^{4} d x \\
& =\frac{4}{3} \pi T \Delta \nu
\end{aligned}
$$

Please note that the signal transfer function $H_{v}(f)$ of the IF filter acts on the signal spectrum and not the phase spectrum. Therefore, the shape of the IF filter signal transfer function $H_{v}(f)$ is not readily calculated. However, as long as the phase-noise modulation index is small, the signal transfer function $H_{v}(f)$ of the filter is identical to the phase transfer function $H_{\phi}(f)$. This aspect is discussed further in Appendix A. Finally, it should also be noted that real signals and filters will deviate from this idealized model for many reasons including practical limitations.

The specifications for carrier phase fluctuations in a microwave link are usually set at the output of the transmitter. However, the spectrum of a phase stable microwave oscillator will not be influenced by the receiver filter duc to the very low amount of phase noise so the same performance would be observed at the demodulator input. This situation is different for an optically generated microwave carrier because of the high amount of phase noise. The overall system performance can be achieved as required even if the transmitter phase noise (7) is slightly too high provided the phase noise at the demodulator input is as specified (8). Therefore, if the transmitter carrier must comply with the specifications for rms differential phase error given by (7) then the beat signal linewidth requirement is

$$
\Delta \nu \leq \frac{1}{4 \pi}\left(\sigma_{\Delta \phi}\right)^{2} R_{b} ; \quad R_{b}=\frac{2}{T}
$$

but if the carrier specifications may be applied after the IF filter at the input of the demodulator then the beat signal linewidth requirement is found from (8)

$$
\Delta \nu \leq \frac{3}{8 \pi}\left(\sigma_{\Delta \phi}\right)^{2} R_{b} ; \quad R_{b}=\frac{2}{T}
$$

where $R_{b}$ is the bit rate for the QPSK signal. The rms differential phase error in the receiver may not exceed $2.8^{\circ}$ 
TABLE I

Total Laser LiNewidth REQuirements IN COHERENT OBFN'S

(c.f. Section III-A). This requirement is given for the rms differential phase error generated by the total amount of phase noise in the transmitter system. Leaving a margii for the rest of the phase-noise sources in the complete OBFN, the maximum rms differential phase error generated by the transmitter module should be within 1-2 degrees. Inserted into (10) this leads to the total laser linewidth requirements listed in Table I.

IV. Improvements by Using Phase-Locked Loops

The requirements for the linewidth of the lasers are not easily fulfilled with free running semiconductor lasers.

\begin{tabular}{ccc}
\hline & \multicolumn{2}{c}{ Required Linewidth in $\mathrm{kHz}$} \\
\cline { 2 - 3 } $\begin{array}{c}\text { QPSK Bit Rate } \\
\mathrm{Mb} / \mathrm{s}\end{array}$ & $\begin{array}{c}{ }^{\circ} \mathrm{rms} \\
\text { Phase Error }\end{array}$ & $\begin{array}{c}2^{\circ} \mathrm{rms} \\
\text { Phase Error }\end{array}$ \\
\hline 33 & 1.2 & 4.8 \\
131 & 4.8 & 19.0 \\
\hline
\end{tabular}

In order to estimate the upper limit of the phase-noise reduction that can be obtained by using a PLL in the transmitter, the maximum theoretical PNR is calculated. $\mathrm{PNR}_{\max }$ is obtained for a loop that removes all the phase noise below the loop bandwidth $f_{z} T$ and is given by

$$
\mathrm{PNR}_{\max }=\frac{\left(\sigma_{\Delta \phi}\right)^{2}}{\left(\sigma_{\Delta \phi}\right)^{2} \text { above } f_{z} T}=\frac{\pi}{3 \int_{\pi f_{z} T}^{\infty}\left(\frac{\sin x}{x}\right)^{4} d x}
$$

\section{B. Phase-Noise Reductions Obtained by the Use of Phase-Locked Loops}

The effect of a frequency or phase-locked loop may be calculated by multiplying the noise spectrum with the square of the modulus of the PLL error function before the calculation of the rms value of the differential phase fluctuations. The modulus squared of the error functions of a first-order PLL and a second-order critical damped PLL are given by (c.f. Appendix B)

$$
|1-H(x)|_{1 . \text { order }}^{2}=\frac{x^{2}}{x^{2}+A^{2}-2 x A \sin \left(x R_{b} \tau_{d}\right)}
$$

$$
\begin{aligned}
& |1-H(x)|_{2 . \text { order }}^{2}= \\
& \quad \frac{x^{4}}{x^{4}+\left(\frac{A}{\sqrt{1+\sqrt{2}}}\right)^{4}+2\left(\frac{A}{\sqrt{1+\sqrt{2}}}\right)^{2} x^{2}\left(1-\cos \left(x R_{b} \tau_{d}\right)\right)-\sqrt{8}\left(\frac{A}{\sqrt{1+\sqrt{2}}}\right) x^{3} \sin \left(x R_{b} \tau_{d}\right)}
\end{aligned}
$$

respectively. The variable $A$ is given by $A=\pi f_{z} T$ and $R_{b} \tau_{d}$ is the normalized loop propagation delay. When phase locking the lasers to each other we find from (8), (14), and (15) the differential phase fluctuations

$$
\left(\sigma_{\Delta \phi}\right)_{\mathrm{PLL}}^{2}=4 T \Delta \nu \int_{0}^{\infty}\left(\frac{\sin x}{x}\right)^{4}|1-H(x)|^{2} d x .
$$

\section{A. Maximum Theoretical Phase-Noise Reduction Limit}

When the two lasers in the transmitter are phase locked to each other, the phase fluctuations of the difference frequency will be modified by the transfer function of the locking loop. We define the phase-noise reduction (PNR) as the ratio between the amount of phase noise when no phase-locked loop (PLL) is present and the amount of phase noise when a PLL with a normalized bandwidth of $f_{z} T$ is used

$$
\text { PNR }=\frac{\text { Phase noise without PLL }}{\text { Phase noise with } \operatorname{PLL}\left(f_{z} T\right)}=\frac{\left(\sigma_{\Delta \phi}\right)^{2}}{\left(\sigma_{\Delta \phi}\right)_{\operatorname{PLL}\left(f_{z} T\right)}^{2}} .
$$

Here $f_{z}$ is the open loop zero decibel frequency as defined in Appendix B and $T$ is the symbol duration. By inserting (8) into (11) we get

$$
\mathrm{PNR}=\frac{4 \pi \Delta \nu T}{3\left(\sigma_{\Delta \phi}\right)_{\mathrm{PLL}\left(f_{i} T\right)}^{2}} .
$$

Inserting (16) into (12) gives the phase-noise reduction achieved (after the receiver IF filter) with PLL's

$$
\text { PNR }=\frac{\pi}{3 \int_{0}^{\infty}\left(\frac{\sin x}{x}\right)^{4}|1-H(x)|^{2} d x}
$$

Numerical integration for different values of $f_{z} T$ then gives the values for the phase-noise reduction PNR of $\left(\sigma_{\Delta \phi}\right)^{2}$ relative to the value for free-running lasers. The $\mathrm{PNR}_{\max }$ and the PNR for ideal first- and second-order loops are shown versus $f_{z} T$ in Fig. 3. It should be noticed that the influence of the loop propagation delay has been neglected (i.e., $\tau_{d}=0$ ). The nonideal situation where $\tau_{d}>$ 0 will be treated in the following section.

From Fig. 3 it can be seen that the first- and secondorder PLL's give almost the same phase-noise reduction for low loop bandwidths. Furthermore, the first-order loop 


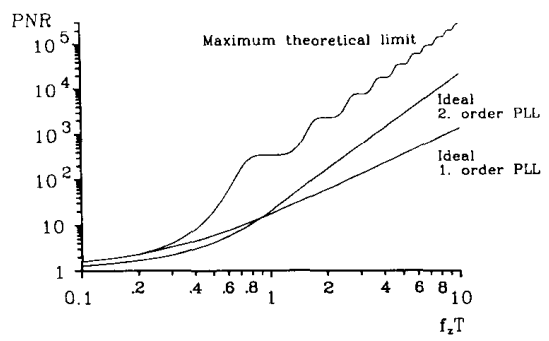

Fig. 3. Phase-noise reduction as a function of normalized loop bandwidth $\left(\tau_{d}=0\right)$.

is slightly better for low loop bandwidths. This is due to the second-order loop's noise enhancement close to $f_{z}$ [32], [33]. The noise enhancement is determined by the damping factor (here $\zeta=1 / \sqrt{2}$, c.f. Appendix B). The larger the damping factor, the closer the PNR of the second-order loop would be to the PNR of the first-order loop both for low and high loop bandwidths. These results indicate that the second-order PLL should only be used in the high bandwidth cases. Furthermore, there is a substantial difference between the maximum theoretical phase-noise reduction limit and the phase-noise reduction obtained using first- and second-order PLL's. This implies that even larger reductions may be obtained using higher order PLL's. Unfortunately, these are even more difficult to implement than the first- and second-order PLL's.

Knowing the phase-noise reduction, it is possible to calculate the beat signal linewidth requirement (sum of laser linewidths) for a given amount of differential phase fluctuations $\left(\sigma_{\Delta \phi}\right)^{2}$ in the receiver when using a PLL in the transmitter module. The requirement is found by multiplying $\Delta \nu$ in (10) by the PNR

$$
\Delta \nu_{\mathrm{PLL}} \leq \Delta \nu \mathrm{PNR}=\frac{3}{8 \pi}\left(\sigma_{\Delta \phi}\right)^{2} R_{b} \mathrm{PNR} .
$$

Even though the largest amount of phase noise is removed by suppressing the phase deviations at the lower frequencies, the results show that the loop bandwidth cannot be much smaller than $1 / T$ for which the phase-noise reduction is approximately 20 . Consequently, PLL's with loop bandwidths $f_{z}$ of $2 / T\left(=R_{b}\right)$ or more are considered to be the best for use in a coherent OBFN transmitter module with semiconductor lasers.

\section{Considerations on Implementation of Phase- LOCKED LOOPS}

Although a phase-locked loop reduces the phase noise significantly at low frequencies, the reduction drops to unity near the frequency of zero decibel open-loop gain, $f_{z}$, and the phase noise above that frequency remains unchanged by the loop. Close to $f_{z}$ the phase noise may be enhanced by the, loop [32], [33] thus making a careful loop design very important. Factors such as propagation delay, PLL phase-error variance $\left(\sigma_{\phi}\right)^{2}$, shot noise, loop bandwidth, and laser linewidth have a large influence on PLL performance as will be discussed in this section.

\section{A. Influence of Loop Propagation Delay}

In Section IV-B the PNR of $\left(\sigma_{\Delta \phi}\right)^{2}$ in the microwave receiver was calculated for ideal first-and second-order loops neglecting the loop propagation delay $\tau_{d}$ (i.e., $\tau_{d}=$ 0 ). However, for broad-band PLL's, the loop propagation delay $\tau_{d}$ has a large influence on loop stability and performance [34]. At the frequency where the phase of the open loop transfer function $G(j \omega)$ (c.f. Appendix B) equals $\pi$

$$
\angle G(j \omega)=-\pi
$$

it is required for stability that [32], [33]

$$
|G(j \omega)|<1 .
$$

Equations (19) and (20) results in the following condition for absolute stability. For a first-order loop we find

$$
\tau_{d}<\frac{\pi}{2 \omega_{z}}=\frac{1}{4 f_{z}}=\frac{0.25}{f_{z}}
$$

and normalization with respect to the bit rate gives

$$
R_{b} \tau_{d}<\frac{1}{2 f_{z} T}=\frac{0.5}{f_{z} T} .
$$

For the second-order described here (c.f. Appendix B) we find, as also derived in [34]

$$
\tau_{d}<\frac{0.736}{\omega_{n}} .
$$

Inserting the natural angular frequency $\omega_{n}$ for the critically damped loop (c.f. Appendix B) gives

$\tau_{d}<\frac{0.736 \sqrt{1+\sqrt{2}}}{\omega_{z}}=\frac{0.736 \sqrt{1+\sqrt{2}}}{2 \pi f_{z}}=\frac{0.182}{f_{z}}$

and normalization with respect to the bit rate gives

$$
R_{b} \tau_{d}<\frac{0.364}{f_{z} T}
$$

Equations (21), (22), (24), and (25) express the limit of $\tau_{d}$ for stable loop operation.

Knowing the limit of $\tau_{d}$ makes it possible to calculate the PNR of $\left(\sigma_{\Delta \phi}\right)^{2}$ in the microwave receiver for different values of $R_{b} \tau_{d}$ using (14), (15), and (17). The results are shown for the first-order loop in Fig. 4 and for the secondorder loop in Fig. 5.

From the curves it is obvious that the loop propagation delay has a large influence on the phase-noise reduction PNR obtained with broad-band PLL's and therefore cannot be neglected. The limits of $\tau_{d}$ are most severe for the second-order loop as seen in Figs. 4 and 5 and from (22) and (25). However, for PNR's greater than 100, the second-order loop may still be the best choice since the loop bandwidth required is smaller than for a first-order loop. 


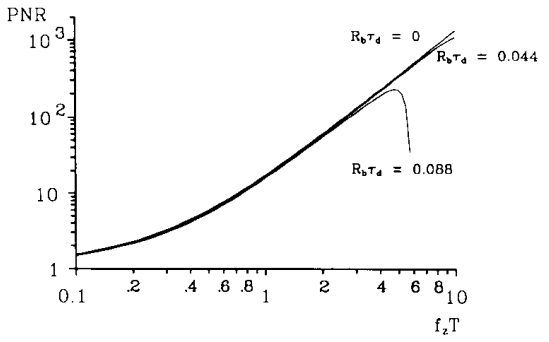

Fig. 4. Phase-noise reduction obtained with a first-order loop as a function of normalized loop bandwidth for different values of normalized time delay.

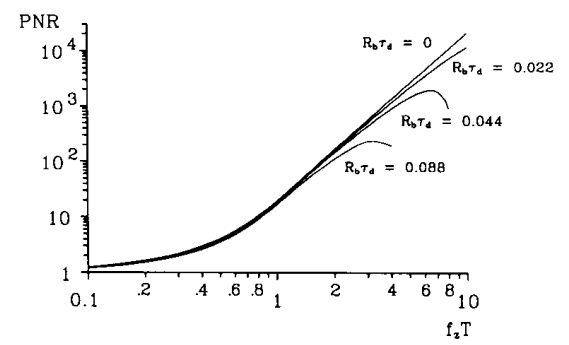

Fig. 5. Phase-noise reduction obtained with a second-order loop as a function of normalized loop bandwidth for different values of normalized time delay.

It should be noted that the point where some of the curves reach a dip at high $f_{z} T$ corresponds to the point of instability. Furthermore, the bandwidth $f_{z} T$ should be kept below the point where the PNR reaches its maximum. Beyond this point, the phase margin in the loop is so small that the probability of unlocking and instability is very large.

The linewidth requirements for a given amount of differential phase fluctuations $\left(\sigma_{\Delta \phi}\right)^{2}$ in the microwave receiver can now be calculated from (18) when using a firstor a second-order PLL in the transmitter module of the coherent OBFN. $\Delta \nu_{\mathrm{PLL}}$ is calculated for a given loop as a function of normalized loop bandwidth $f_{z} T$ and normalized loop propagation delay $R_{b} \tau_{d}$. fluctuations $\left(\sigma_{\Delta \phi}\right)^{2}$, the total laser linewidth has to be be-
In addition to the requirements for the differential phase

also by the frequency translated shot noise [35]. Assuming linear PLL analysis as in this paper the $\left(\sigma_{\phi}\right)^{2}$ is given by [35]

$$
\begin{aligned}
\left(\sigma_{\phi}\right)^{2}= & \int_{0}^{\infty} S_{\phi}(f)|1-H(j 2 \pi f)|^{2} d f \\
& +\int_{0}^{\infty} \frac{S_{q}(f)}{K_{1}^{2}}|H(j 2 \pi f)|^{2} d f \leq 0.25
\end{aligned}
$$

where $H(j 2 \pi f)$ is the PLL transfer function and $1-$ $H(j 2 \pi f)$ is the PLL error function (c.f. Appendix B). $S_{\phi}(f)$ is the single sideband phase fluctuation spectrum, $S_{q}(f)$ is the single sideband quantum shot-noise spectrum and $K_{1}$ is the conversion gain of the front end and the phase detector in the PLL (c.f. Fig. 7). However, since the optical input power is large in a coherent OBFN PLL transmitter module the shot noise will have negligible effect on the phase-error variance which is then given by

$$
\left(\sigma_{\phi}\right)^{2}=\frac{\Delta \nu}{\pi} \int_{0}^{\infty} \frac{|1-H(j 2 \pi f)|^{2}}{f^{2}} d f \leq 0.25 .
$$

Although not shown explicitly, the linear analysis is valid for the calculations in this paper. For the laser linewidths considered (order of megahertz) $\left(\sigma_{\phi}\right)^{2}$ is well below 0.25 but even though $\left(\sigma_{\phi}\right)^{2}$ is small there is still a finite probability for loss of lock.

\section{Linewidth Requirements for Stable Locked Loop Operation}

Even though the PLL operates in the linear region a finite probability exists that a noise-induced phase excursion will throw the loop out of lock causing the VCO laser to slip one or more cycles. The average time between such events, assuming zero mean phase error, can be approximated by [32], [33], [36]

$$
T_{A V} \cong \frac{\pi e^{2 /\left(\sigma_{\phi}\right)^{2}}}{4 \int_{0}^{\infty}|H(j 2 \pi f)|^{2} d f}
$$

Inserting (27) into (28) and separating with respect to the beat signal linewidth $\Delta \nu$ we find

$$
\Delta \nu \leq \frac{2 \pi}{\int_{0}^{\infty} \frac{|1-H(j 2 \pi f)|^{2}}{f^{2}} d f \cdot \ln \left(\frac{4 T_{A V}}{\pi} \int_{0}^{\infty}|H(j 2 \pi f)|^{2} d f\right)}
$$

low a certain value to ensure stable locked loop operation. In other words, the phase-error variance $\left(\sigma_{\phi}\right)^{2}$ in the loop has to be low enough to ensure stable-locked loop operation.

\section{B. PLL Phase-Error Variance and Shot-Noise Influence}

The phase-error variance $\left(\sigma_{\phi}\right)^{2}$ in an optical PLL (c.f.

Fig. 7, Appendix B) is determined not only by the laser
This equation can be used to calculate the maximum allowable total laser linewidth for a given average time to cycle slip $T_{A V}$, a given loop propagation delay $\tau_{d}$, and a given loop bandwidth $f_{z}$.

Using the expressions for the transfer function and the error function given in Appendix B we have calculated $\Delta \nu$ by numerical integration. The results are shown in Fig. 6 for a second-order loop since this is considered the most promising. An average time to cycle slip of $T_{A V}=10$ 


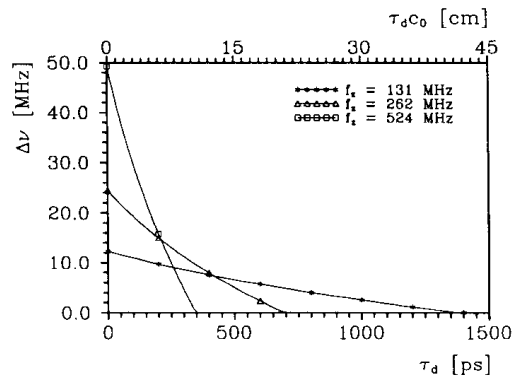

Fig. 6. Laser linewidth demand for stable locked loop operation as a function of propagation delay and loop bandwidth for an average time to cycle slip of 10 years.

years was selected, a value sufficient to give reliable operation in practical systems. The demands for laser linewidth given by (31) would be less demanding for the firstorder loop.

We now have two linewidth requirements. The first requirement, which is given by (18) and the PNR in Fig. 5, yields the maximum linewidth allowable to obtain a given amount of differential phase fluctuations $\left(\sigma_{\Delta \phi}\right)^{2}$ in the earth station microwave DQPSK receiver. The second requirement, which is given by (31) and Fig. 6, yields the maximum linewidth allowable to obtain stable locked loop operation with a given average time to cycle slip $T_{A V}$, i.e., a phase-error variance $\left(\sigma_{\phi}\right)^{2}$ in the PLL that is small enough. The total linewidth of the lasers in the PLL transmitter module has to fulfill the most restrictive of the two requirements mentioned above.

\section{A Numerical Example}

The use of a PLL in the transmitter module of the coherent OBFN makes it possible to fulfill the demands placed on $\left(\sigma_{\Delta \phi}\right)^{2}$ and thereby on the sum of the laser linewidths. The linewidths required when using phase-locked loops are within the range of linewidths reported for semiconductor lasers. For DFB lasers, linewidths of approximately $1 \mathrm{MHz}$ are state of the art [37] and linewidths as low as $250 \mathrm{kHz}$ have been reported [38]. For multielectrode DFB lasers with uniform frequency response, linewidths in the order of a few megahertz have been reported [39]-[41].

For the system used as an example here, we focus on the high $(131 \mathrm{Mb} / \mathrm{s})$ data rate. The rms differential phase error $\sigma_{\Delta \phi}$ should be within $1-2^{\circ}$ and the system has to be implemented with existing lasers, i.e., lasers with linewidths in the order of megahertz. From the linewidth requirements in Table I and (18) we find that a PNR of around 1000 is needed to fulfill the demands. Looking at Fig. 3, a broad-band second-order PLL seems the most obvious choice in order to obtain the needed PNR. Subsequently, from Fig. 5, we may choose a loop bandwidth of $f_{z} T=4\left(f_{z}=262 \mathrm{MHz}\right)$. Allowing for a loop propagation delay of $R_{b} \tau_{d}=0.044\left(\tau_{d}=330 \mathrm{ps}, \tau_{d} c_{0}=10\right.$ $\mathrm{cm}$ ) gives a PNR $=890$ whereby the phase-noise requirements can be fulfilled using lasers with linewidths in the order of a few megahertz. Calculating the linewidth requirement from (18) we find that the total laser linewidth should be below $4.2 \mathrm{MHz}$ to obtain $\sigma_{\Delta \phi}=1^{\circ}$ and below $17 \mathrm{MHz}$ to obtain $\sigma_{\Delta \phi}=2^{\circ}$. Finally, it is necessary to ensure that the PLL operates as a stable locked loop. From Fig. 6 we see that for $f_{z}=262 \mathrm{MHz}$ and $\tau_{d} c_{0}=10 \mathrm{~cm}$, the total linewidth should be within $10 \mathrm{MHz}$ which will result in $\sigma_{\Delta \phi}=1.5^{\circ}$. The above calculations show that the maximum total laser linewidth should be within 4-10 $\mathrm{MHz}$, permitting a maximum linewidth within $2-5 \mathrm{MHz}$ for each laser.

The implementation of the PLL is challenging since the physical (free space) length of the loop's electronic and optical signal path could be no longer than $10 \mathrm{~cm}$. To ensure a stable operation of the PLL, it is therefore necessary to use microoptics and small electronic circuitry with very short time delays.

\section{Conclusion}

The principles and advantages of a coherent OBFN for use in satellites with active phased array antennas has been outlined together with a power budget estimation for the OBFN.

Based on the assumption of a white frequency fluctuation spectrum for the lasers, the rms differential phase fluctuations at the demodulator of a DQPSK microwave receiver have been calculated when fed from such a system. From this, the requirement for laser linewidth has been deduced for QPSK modulation schemes to fulfill specifications for rms differential phase errors.

The requirements for laser linewidth prove very difficult to meet with existing free-running semiconductor lasers. However, the requirements are significantly reduced by stabilizing the frequency difference between the two lasers of the transmitter module by the use of PLL's, and the phase-noise reduction has been calculated taking the loop propagation delay into account. In addition, the influence of shot noise has been evaluated and a criterion for stable locked loop operation has been derived. It was shown that a PLL enables the use of lasers with linewidths of up to a few megahertz. Such lasers have been reported and may be commercially available in the near future.

In conclusion; coherent OBFN's seem a realistic approach to beam forming in future advanced satellite communication systems with active phased array antennas. They show a far better power budget than other types of OBFN's and the use of coherent optical techniques open possibilities for effective optical control and optical processing. Optical techniques provide the advantage of low weight, small size, flexibility, and immunity to electromagnetic interference and finally, optical techniques have the potential of optoelectronic integration, which eventually will result in very compact and low-cost systems. 
APPENDiX A

Evaluation of the IF Filter Signal Transfer FUNCTION

The relation between the $\sin (x) / x$ phase transfer function $H_{\phi}(f)$ and the signal transfer function $H_{v}(f)$ of the IF filter is very difficult to calculate by exact analysis. It is, however, possible to evaluate the signal transfer function by approximation.

The phase-noise power spectrum $S_{\phi}(f)$ and the signal power spectrum $\mathscr{L}(f)$ after the filter are calculated as

$$
\begin{gathered}
S_{\phi}(f)_{2}=\left|H_{\phi}(f)\right|^{2} \cdot S_{\phi}(f)_{1} \\
\mathcal{L}(f)_{2}=\left|H_{v}(f)\right|^{2} \cdot \mathcal{L}(f)_{1}
\end{gathered}
$$

where indexes 2 and 1 refer to the spectra after and before the IF filter, respectively. Provided that the phase-noise modulation index is small, i.e., $\phi_{\text {peak }} \ll 1$, then the phase-noise spectrum and the signal power spectrum is related as [42]

$$
\mathcal{L}(f)=\frac{1}{2} \cdot S_{\phi}(f) .
$$

Therefore, at small modulation index the signal transfer function may be found as

$$
\left|H_{v}(f)\right|^{2}=\left|H_{\phi}(f)\right|^{2}, \quad \text { for } \phi_{\text {peak }} \ll 1 .
$$

This situation is not fulfilled close to the carrier frequency of the IF signal. The signal transfer function will, therefore, be different from the phase transfer function at offset frequencies close to the carrier frequency. However, this has no importance in the situations where the filter is so broad that it does not limit the carrier spectrum, i.e., when $\Delta \nu \ll B_{\mathrm{IF}}$.

Other authors have performed analysis in the time domain for an IF filter with a $\sin (x) / x$ signal transfer function [43]. Their analysis have shown, that such an IF filter alters the differential phase fluctuations (of free-running lasers) as

$$
\left(\sigma_{\Delta \phi}\right)_{2}^{2}=a \cdot\left(\sigma_{\Delta \phi}\right)_{1}^{2}
$$

where $a=0.726$ was calculated for linewidths of up to $5 \%$ of the filter bandwidth. In our calculations we have used an IF filter with a $\sin (x) / x$ phase transfer function and $a=0.667$ was found (comparing (7) and (8)). So even though the demand for $\phi_{\text {peak }} \ll 1$ may not be fulfilled there is no large difference between the results. Hence, in the situation where the filter is fairly broad compared to the carrier spectrum (A4) may be used as a fairly good approximation for evaluating the signal transfer function.

\section{APPENDIX B}

Phase-Locked Loop Transfer and Error Functions

A phase-locked loop (PLL) (c.f. Fig. 7) taking loop propagation delay into account is normally described by

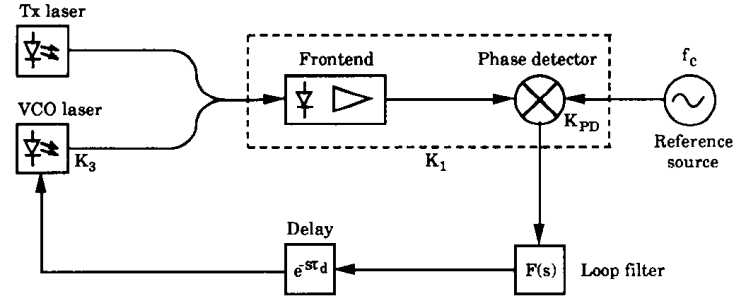

Fig. 7. Coherent OBFN phase-locked loop transmitter module

the parameters [32], [33]

Open loop transfer function: $G(s)$

$$
=\frac{K_{1} K_{3} F(s) e^{-s \tau_{d}}}{s}=\frac{K F(s) e^{-s \tau_{d}}}{s}
$$

Closed loop transfer function: $H(s)$

$$
=\frac{G(s)}{1+G(s)}=\frac{K F(s) e^{-s \tau_{d}}}{s+K F(s) e^{-s \tau_{d}}}
$$

Error function: $1-H(s)$

$$
=\frac{1}{1+G(s)}=\frac{s}{s+K F(s) e^{-s \tau_{d}}}
$$

when assuming linear stable locked loop operation; a condition which, as shown in Section V, is fulfilled. For real frequencies $s=j \omega=j 2 \pi f . K_{1}$ is the conversion gain of the front end and the phase detector, $K_{3}$ is the voltage controlled oscillator (VCO) laser modulation sensitivity, $F(s)$ is the transfer function of the loop filter, $\tau_{d}$ is the loop propagation delay, and $K$ is the open loop gain of the phase-locked loop. A factor $K_{2}$ can be introduced when an amplifier is present between the loop filter and the VCO laser. The error function of the loop is given as the phase error divided by the phase fluctuations of the phase detector input signal. So the error function is an expression of the noise reduction that can be obtained on the input signal when using a PLL.

\section{A. First-Order Loop}

A first-order loop has $F(s)=1$ and may be implemented with a frequency detector and an integrator instead of a phase detector. We have the error function from (B3)

$$
\begin{aligned}
1-H(j \omega) & =\frac{j \omega}{j \omega+K e^{-j \omega \tau_{d}}}=\frac{j \omega}{j \omega+\omega_{z} e^{-j \omega \tau_{d}}} \\
|1-H(j \omega)|^{2} & =\frac{\omega^{2}}{\omega^{2}+\omega_{z}^{2}-2 \omega \omega_{z} \sin \left(\omega \tau_{d}\right)}
\end{aligned}
$$

where the open-loop gain $K$ is equal to the angular frequency of zero decibel loop gain called $\omega_{z}$. Introducing $x$ $=\pi f T, A=\pi f_{z} T$ and the normalized loop propagation delay $R_{b} \tau_{d}\left(R_{b}=2 / T\right)$ to allow for the same frequency 
normalization as that used for $\sin (x) / x$ in (7) we get

$$
|1-H(x)|^{2}=\frac{x^{2}}{x^{2}+A^{2}-2 x A \sin \left(x R_{b} \tau_{d}\right)} .
$$

The open-loop transfer function of the first-order loop is given by (B1)

$$
\begin{aligned}
G(j \omega) & =\frac{K e^{-j \omega \tau_{d}}}{j \omega} \\
\left|G\left(j \omega_{z}\right)\right| & =1, \quad \text { for } K=\omega_{z}
\end{aligned}
$$

The transfer function of the active filter is given by [32], [33]

$$
F(s)=\frac{s \tau_{2}+1}{s \tau_{1}}
$$

From (B3) and (B11) we get the error function

$$
1-H(j \omega)=\frac{-\omega^{2}}{-\omega^{2}+\omega_{n}^{2} e^{-j \omega \tau_{d}}+j 2 \zeta \omega_{n} \omega e^{-j \omega \tau_{d}}}
$$

$$
|1-H(j \omega)|^{2}=\frac{\omega^{4}}{\omega^{4}+\omega_{n}^{4}+\omega_{n}^{2} \omega^{2}\left(4 \zeta^{2}-2 \cos \left(\omega \tau_{d}\right)\right)-4 \zeta \omega_{n} \omega^{3} \sin \left(\omega \tau_{d}\right)}
$$

$$
\angle G(j \omega)=-\frac{\pi}{2}-\omega \tau_{d}
$$

where $\omega_{n}$ and $\zeta$ are the natural frequency and the damping coefficient of the loop given by [32], [33]

$$
\omega_{n}=\sqrt{\frac{K}{\tau_{1}}}, \quad \zeta=\frac{\omega_{n} \tau_{2}}{2}
$$

In order to compare the second-order loop to the first-order loop, it is convenient to normalize with respect to the frequency of zero decibel open-loop gain called $\omega_{z}$. The open-loop transfer function is given by (B1)

$$
\begin{aligned}
G(j \omega) & =-\frac{\omega_{n}^{2}}{\omega^{2}}\left(1+2 \zeta \frac{j \omega}{\omega_{n}}\right) e^{-j \omega \tau_{d}} \\
\left|G\left(j \omega_{z}\right)\right| & =1, \quad \text { for }\left(\frac{\omega_{z}}{\omega_{n}}\right)^{2}=2 \zeta^{2}+\sqrt{4 \zeta^{4}+1} \\
\omega_{n} & =\frac{\omega_{z}}{\sqrt{2 \zeta^{2}+\sqrt{4 \zeta^{4}+1}}} \\
\angle G(j \omega) & =\arctan \frac{\omega \tau_{2} \cos \left(\omega \tau_{d}\right)-\sin \left(\omega \tau_{d}\right)}{\cos \left(\omega \tau_{d}\right)+\omega \tau_{2} \sin \left(\omega \tau_{d}\right)} .
\end{aligned}
$$

Introducing $x=\pi f T, A=\pi f_{z} T$ and the normalized loop propagation delay $R_{b} \tau_{d}\left(R_{b}=2 / T\right)$ and get from (B13) and (B17) for a critically damped loop which is achieved for $\zeta=0.707$ [32], [33]

$$
|1-H(x)|^{2}=\frac{x^{4}}{x^{4}+\left(\frac{A}{\sqrt{1+\sqrt{2}}}\right)^{4}+2\left(\frac{A}{\sqrt{1+\sqrt{2}}}\right)^{2} x^{2}\left(1-\cos \left(x R_{b} \tau_{d}\right)\right)-\sqrt{8}\left(\frac{A}{\sqrt{1+\sqrt{2}}}\right) x^{3} \sin \left(x R_{b} \tau_{d}\right)} .
$$

passive filter, although the phase-noise reduction obtained with the active filter is slightly higher than the phase-noise reduction obtained with the passive filter.
Furthermore, we may note that with the normalization used both first- and second-order loops have open-loop zero decibel gain for $x=A$. 
Finally, the transfer function of the second-order loop is given by $(\mathrm{B} 2)$

$$
\begin{aligned}
& H(j \omega)=\frac{\omega_{n}^{2} e^{-j \omega \tau_{d}}+j 2 \zeta \omega_{n} \omega e^{-j \omega \tau_{d}}}{-\omega^{2}+\omega_{n}^{2} e^{-j \omega \tau_{d}}+j 2 \zeta \omega_{n} \omega e^{-j \omega \tau_{d}}} \\
& |H(j \omega)|^{2}=\frac{\omega_{n}^{4}+4 \zeta^{2} \omega_{n}^{2} \omega^{2}}{\omega^{4}+\omega_{n}^{4}+\omega_{n}^{2} \omega^{2}\left(4 \zeta^{2}-2 \cos \left(\omega \tau_{d}\right)\right)-4 \zeta \omega_{n} \omega^{3} \sin \left(\omega \tau_{d}\right)} .
\end{aligned}
$$

ments for optical PSK heterodyne communication systems, " J. Lightwave Technol., vol. LT-4, no. 4, pp. 415-425, 1986

[21] G. Jacobsen and I. Garrett, "Theory for optical heterodyne DPSK receivers with post-detection filtering," J. Lightwave Technol., vol.

[22] G. Jacobsen and I. Garrett, "The effect of laser linewidth on coherent

\section{ACKNOWLEDGMENT}

S. N. Madsen and J. Dall are acknowledged for contributing with fruitful suggestions as is L. Eskildsen for assistance on the implementation of the programs for the numerical calculations. Finally, C. Mahon is acknowledged for valuable comments on the manuscript.

\section{REFERENCES}

[1] J. E. Bowers, "Optical transmission using PSK-modulated subcarriers at frequencies to $16 \mathrm{GHz}, "$ Electron. Lett., vol. 22, no. 21, pp. $1119-1121,1986$

[2] J. E. Bowers, A. C. Chipaloski, S. Boodaghians, and J. W. Carlin, "Direct fibre-optic transmission of entire microwave satellite antenna signals," Electron. Lett., vol. 23, no. 5, pp. 185-187, 1987.

[3] J. E. Bowers, A. C. Chipaloski, S. Boodaghians, and J. W. Carlin, "Long distance fiber-optic transmission of $C$-band microwave signal to and from satellite antenna," J. Lightwave Technol., vol. LT-5, no. 12 , pp. $1733-1741,1987$.

[4] A. Daryoush et al., "Optical beam control of mm-wave phased array antennas for communications," Microwave J., vol. 30, no. 3, pp. 97-104, 1987.

[5] W. Ng, A. Walston, G. Tangonan, J. J. Lee, and I. Newberg, "Op tical steering of dual and band microwave phased array antenna using semiconductor laser switching," Electron. Lett., vol. 26, no. 12, pp. 791-793, 1990.

[6] R. Benjamin, C. D. Zaglanikis, and A. J. Seeds, "Optical beam former for phased arrays with independent control of radiated frequency and phase," Electron. Lett., vol. 26, no. 22, pp. 1853-1855, 1990.

[7] L. Goldberg, H. F. Taylor, and J. F. Weller, "Microwave signal generation with injection-locked laser diodes," Electron. Lett., vol. 19 , no. 13, pp. 491-493, 1983

[8] L. Goldberg, A. M. Yurek, H. F. Taylor, and J. F. Weller, "' $35 \mathrm{GHz}$ microwave signal generation with an injection-locked laser diode," Electron. Lett., vol. 21, no. 18, pp. 814-815, 1985.

[9] M. Tamburrini, M. Parent, L. Goldberg, and D. Stillwell, "Optical feed for a phased array microwave antenna," Electron. Lett., vol. 23 , по. 13 , pp. $680-681,1987$

[10] G. L. Abbas and S. Thaniyavarn, "Millimeter wave generation and phase control using optical heterodyne techniques and waveguide de vices," in Dig. Conf. Optical Fiber Commun. (San Fransisco, CA), 1990 Technical Digest Series, vol. 1 (Optical Society of America, Washington, DC 1990), pp. 150-151.

[11] E. N. Toughlian, H. Zmuda, and P. Kornreich, "A deformable mir ror-based optical beamforming system for phased array antennas," IEEE Photon. Technol. Lett., vol. 2, no. 6. pp. 444-446, 1990

[12] Intelsat earth station standards (IESS), Document IESS-309, 1990.

[13] R. C. Steele, "Optical phase-locked loop using semiconductor lase diodes," Electron. Lett., vol. 19, no. 2, pp. 69-70, 1983.

[14] J. Harrison and A. Mooradian, "Spectral linewidth of semiconducto lasers," in Methods of Laser Spectroscopy, Y. Prior, Ed. New York: Plenum, 1986, pp. 133-142.

[15] M. Ohtsu, "Realization of ultrahigh coherence in semiconductor lasers by negative electrical feedback," J. Lightwave Technol., vol. 6 , no. 2, pp. 245-256, 1988.

[16] P. Balling, K. Stubkjær, E. L. Christensen, L. A. Pedersen, and S. Dahl-Petersen, "Optical technologies for beam forming networks," Final Rep. ESTEC Contract 7685/87/NL/JG (SC), 1989.

[17] K. Kikuchi, T. Okoshi, M. Nagamatsu, and N. Henmi, "Degradation of bit-error rate in coherent optical communications due to spectral spread of the transmitter and the local oscillator," J. Lightwave Technol., vol. LT-2, no. 6, pp. 1024-1033, 1984.

[18] L. G. Kazovsky, "Impact of laser phase noise on optical heterodyne communication systems," J. Optical Comm., vol. 7, no. 2, pp. 66$78,1986$.

[19] B. Glance, "Performance of homodyne detection of binary PSK optical signals," J. Lightwave Technol., vol. LT-4, no. 2, pp. 228-235, 1986.

[20] L. G. Kazovsky, "Performance analysis and laser linewidth requireoptical receivers with nonsynchronous demodulation," J. Lightwave Technol., vol. LT-5, no. 4, pp. 551-560, 1987.

[23] T. G. Hodgkinson, "Receiver analysis for synchronous coherent optical fiber transmission systems," $J$. Lightwave Technol., vol. LT-4, no. 2, pp. 573-586, 1986.

[24] E. Patzak and P. Meissner, "Influence of IF-filtering on bit error rate floor in coherent optical DPSK-systems." IEE Proc. $J$., vol. 135, no. 5. pp. 355-357, 1988.

125] H. E. Rowe, Signals and Noise in Communication Systems. New York: Van Nostrand, 1965, pp. 113-116

[26] B. Daino, P. Spano, M. Tamburrini, and S. Piazzolla, "Phase noise and spectral line shape in semiconductor lasers," IEEE J. Quantum Electron., vol. QE-19, no. 3, pp. 266-270, 1983

[27] P. Spano, S. Piazzolla, and M. Tamburrini, "Phase noise in semiconductor lasers: A theoretical approach," IEEE J. Quantum Electron., vol. QE-19, no. 7, pp. 1195-1199, 1983.

[28] K. Kikuchi and T. Okoshi, "FM- and AM-noise spectra of $1.3 \mu \mathrm{m}$ InGaAsP DFB lasers in $0-3 \mathrm{GHz}$ range and determination of their linewidth enhancement factor $\alpha, "$ Electron. Lett., vol. 20, no. 25/ 26, pp. 1044-1045, 1984.

[29] T. Okoshi and K. Kikuchi. Coherent Optical Fiber Communications. Tokyo, Japan: KTK Scientific, 1988, ch. 3.

[30] I. S. Gradshteyn and I. M. Ryzhik, Table of Integrals, Series, and Products, 4th ed. New York and London: Academic, 1965, seventh printing, 1973, pp. 446-450.

131] K. S. Shanmugam, Digital and Analog Communication Systems New York: Wiley, 1979, ch. 8

[32] F. M. Gardner, Phaselock Techniques. New York: Wiley, 1966

[33] A. Blanchard, Phase-Locked Loops Application to Coherent Receiver Design. New York: Wiley, 1976.

[34] M. A. Grant, W. C. Michie, and M. J. Fletcher, "The performance of optical phase-locked loops in the presence of nonnegligible loop propagation delay," J. Lightwave Technol., vol. LT-5, no. 4, pp. 592-597, 1987.

[35] T. G. Hodgkinson, "Phase-locked loop analysis for pilot carrier coherent optical receivers," Electron. Lett., vol. 21, no. 25/26, pp. $1202-1203,1985$

[36] R. T. Ramos and A. J. Seeds, "Delay, linewidth and bandwidth limitations in optical phase-locked loop design," Electron. Lett., vol. 26, no. 6, pp. 389-391, 1990.

[37] K. Uomi et al., "Spectral linewidth reduction by the spatial hole burning in $1.5 \mu \mathrm{m}$ multi-quantum-well $\lambda / 4$-shifted DFB lasers," Electron. Lett., vol. 26, no. 1, pp. 52-53, 1990.

[38] H. Yamazaki, T. Sasaki, N. Kida, M. Kitamura, and I. Mito, " 250 $\mathrm{kHz}$ linewidth operation in long cavity $1.5 \mu \mathrm{m}$ multiple quantum well DFB-LDs with reduced linewidth enhancement factor," in Dig. Conf. Optical Fiber Commun. (San Fransisco, CA), 1990, post deadline, paper PD33

[39] S. Ogita et al., "FM response of narrow-linewidth, multielectrode $\lambda / 4$ shift DFB laser," IEEE Photon. Technol. Lett., vol. 2, no. 3, pp. 165-166, 1990.

140] Y. Twu et al., "Long-cavity, multi-electrode DFB lasers for coherent FSK systems," Electron. Lett., vol. 26, no. 11, pp. 708-710, 1990.

[41] R. J. S. Pedersen, U. Gliese, B. Broberg, and S. Nilsson, "Characterization of a $1.5 \mu \mathrm{m}$ three-electrode DFB laser," in Proc. European Conf. Optical Commun. (Amsterdam, The Netherlands), 1990, paper TuF3.3

[42] "Phase noise," Hewlett Packard RF and microwave phase noise measurement seminar, Hewlett Packard Publication no.: 5955-8136.

[43] G. Jacobsen, B. Jensen, I. Garrett, and J. B. Waite, "Bit error rate 
floors in coherent optical systems with delay demodulation," Electron. Lett., vol. 25, no. 21 , pp. 1425-1427, 1989.

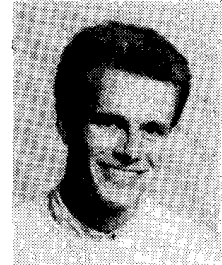

Ulrik Gliese was born in Copenhagen, Denmark, on July 29 , 1965. In 1989 he received the M Sc. degree in electrical engineering from the Electromagnetics Institute, Technical University of Denmark. He is presently pursuing the Ph.D. degre at the Technical University of Denmark with $\mathrm{co}^{-}$ herent optical beam forming networks in satellites as the main research topic.

Since 1989 he has been working as a Research Associate at the Electromagnetics Institute. Hi research activities have covered the design, construction, and characterization of high bit-rate coherent optical communication systems.

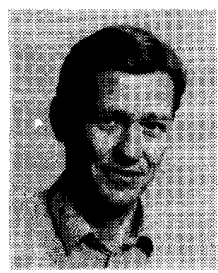

Erik Lintz Christensen received the M.Sc.E.E. degree in 1966

In 1968 he joined the Electromagnetics Institute, Technical University of Denmark, where he is now an Associate Professor. His work has covered many aspects of radar, radio communications, and high frequency and microwave electronics. This includes the design of measurement systems such as 60 - and $300-\mathrm{MHz}$ radars for recording of the thickness of the inland ice of Greenland and Antarctica, equipment for measur- ing amplitude and phase of the transfer function of a $50-\mathrm{km}$ line-of-sight radio path at $15 \mathrm{GHz}$, a system for measuring amplitude and phase of antenna near-field patterns, etc. He is now Project Manager of the Danish Airborne SAR program.

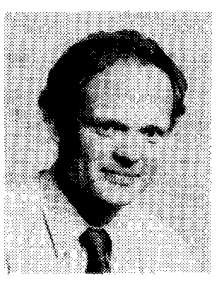

Kristian E. Stubkjaer (S'76-M'81) was born in Aarhus, Denmark, in 1953. He received the M.Sc. and Ph.D. degrees from the Technical University of Denmark, Copenhagen, in 1977 and 1981, respectively. From 1979 to 1981 he studied at the Tokyo Institute of Technology, Tokyo, Japan, with a scholarship from the Japanese Govern ment.

From 1981 to 1982 he was drafted for military service at the Danish Defence Research Establishment in Copenhagen. From 1982 to 1983 he was a Visiting Scientist at the IBM T. J. Watson Research Center, Yorktown Heights, NY. He is now an Associate Professor at the Electromagnetics Institute, Technical University of Denmark, Lyngby, Denmark, where he is working in the field of optical communication. From June 1985 to February 1990 he was Director of the Electromagnetics Institute. 